\title{
A SECOND ORDER ACCURATE KINETIC RELAXATION SCHEME FOR INVISCID COMPRESSIBLE FLOWS
}

\author{
K. R. ARUN, M. LUKÁČOVÁ-MEDVIĎOVÁ, PHOOLAN PRASAD, AND S. V. RAGHURAMA RAO
}

\begin{abstract}
In this paper we present a kinetic relaxation scheme for the Euler equations of gas dynamics in one space dimension. The method is easily applicable to solve any complex system of conservation laws. The numerical scheme is based on a relaxation approximation for conservation laws viewed as a discrete velocity model of the Boltzmann equation of kinetic theory. The discrete kinetic equation is solved by a splitting method consisting of a convection phase and a collision phase. The convection phase involves only the solution of linear transport equations and the collision phase instantaneously relaxes the distribution function to an equilibrium distribution. We prove that the first order accurate method is conservative, preserves the positivity of mass density and pressure and entropy stable. An anti-diffusive Chapman-Enskog distribution is used to derive a second order accurate method. The results of numerical experiments on some benchmark problems confirm the efficiency and robustness of the proposed scheme.
\end{abstract}

\section{INTRODUCTION}

Over the past few decades, the intense research on shock capturing schemes has lead to the development of several numerical methods for the compressible Euler equations of gas dynamics. Of the various methods developed so far, the finite volume methods have been the most popular. The main advantages of the finite volume methods are the simplicity of the scheme and automatic control of conservation, which is a crucial property. These methods can be broadly classified into two categories: central schemes and upwind schemes.

The central schemes originated as the central finite difference formulation of conservation laws. Some prototypes of these schemes are the Lax-Friedrichs scheme and the Lax-Wendroff scheme. The central schemes are less dependent on the eigen-structure of the conservation laws and hence they can also be used for solving non-strictly hyperbolic problems, convection-diffusion problems, etc. In recent years, the central schemes have gained a lot of renewed interest due to their new interpretation as Godunov type schemes on staggered grids. This idea is due to Nessyahu and Tadmor [27]. We refer the reader to [42] for a review of central schemes.

Upwind methods include Riemann solvers (exact and approximate), flux splitting methods, etc. Most of these schemes are based on the hyperbolic structure of the underlying conservation laws. Reviews of upwind methods can be found in the text books by Godlewski and Raviart [16], Hirsch [17], LeVeque [23] and Toro [43]. Another important category of upwind methods are the kinetic schemes. They are based on the Boltzmann equation of kinetic theory which describes the spatialtemporal evolution of the particle density function. The kinetic schemes exploit the fact that nonlinear conservation laws can be recovered by taking various moments of the Boltzmann equation. We refer the reader to the text books by Cercignani [6], Cercignani, Illner and Pulvirenti [7] for a comprehensive treatment of kinetic theory. One of the most fascinating aspects of the kinetic

Date: May 3, 2010.

2000 Mathematics Subject Classification. Primary 35L45, 35L60, 35L65, 35L67; Secondary 65M06, 76P05, 82B40.

Key words and phrases. relaxation systems, hyperbolic conservation laws, discrete velocity Boltzmann equation, Maxwellian, anti-diffusive Chapman-Enskog distribution. 
schemes is that when applied to Euler equations of gas dynamics, they preserve the positivity of mass density and pressure. As a result, the kinetic schemes are unconditionally stable in the $L^{1}$ norm. Further, they also possess the entropy property as a consequence of the celebrated Boltzmann $H$-theorem. For more details of kinetic schemes, see Sanders and Prendergast [39], Pullin [34], Deshpande [11, 12, 13], Godlewski and Raviart [16], Perthame [31, 32] and Xu [46, 47].

Recently, Jin and Xin [20] introduced a new category of upwind methods called relaxation schemes based on the relaxation approximation of conservation laws. In this method, the given nonlinear system of conservation laws is replaced by a larger semi-linear system, known as the relaxation system. The relaxation system has a stiff source term containing a small relaxation parameter $\epsilon$. The original system of conservation laws can be recovered from the relaxation system in the limit as $\epsilon \rightarrow 0$. In [20] Jin and Xin have developed a variety of numerical schemes which are classified into two categories: relaxing schemes and relaxed schemes. The relaxing schemes are obtained by directly discretising the relaxation system and hence they contain the stiff parameter $\epsilon$ explicitly. A relaxed scheme is the limit of a relaxing scheme when $\epsilon=0$. Due to the presence of $\epsilon$, it is in general difficult to attain high order time accuracy in relaxing schemes. However, special Runge-Kutta time stepping schemes have been proposed in $[19,20,29]$ to develop high order relaxation schemes with MUSCL or WENO type space discretisations. It is interesting to note that the diagonal form of a Jin-Xin type relaxation system can be interpreted as a discrete velocity Boltzmann equation $[1,4,33]$. In the literature, lots of numerical studies have been reported in the context of both discrete Boltzmann and relaxation models, see [1, 2, 10, 20, 21, 29, 30, 36, 38, 44] and the references therein.

The goal of the present work is to develop a relaxation scheme for the compressible Euler equations in one space dimension based on a discrete velocity Boltzmann equation. The main advantages of the discrete Boltzmann model are the linearity of the convective part, simplicity compared to classical Boltzmann equation, the diagonal form and the ease for upwinding. Further, we can exploit the vast literature of kinetic theory to design and study numerical schemes based on such discrete kinetic models. We solve the discrete Boltzmann equation by a splitting method consisting of a convection phase and a collision phase. The convection phase involves only the solution of linear transport equations and the collision phase instantaneously relaxes the distribution function to an equilibrium distribution. However, as remarked in [19], such a simple splitting strategy reduces the resulting numerical scheme to formally first order accurate in time. Moreover, the first order scheme suffers from a large amount of numerical dissipation. Nonetheless, in the context of classical kinetic schemes, Deshpande [12] has circumvented these difficulties by the use of an anti-diffusive Chapman-Enskog distribution instead of the Maxwellian. Recently, Kunik et al. [22] employed same mechanism to design a second order kinetic scheme for the relativistic hydrodynamics equations. Following [12, 22] we derive an anti-diffusive Chapman-Enskog distribution for the discrete Boltzmann equation to develop a second order upwind relaxation scheme. It is to be remarked that the Chapman-Enskog method is always associated with nonlinear convection-diffusion equations $[6,7,8]$ and the use of Chapman-Enskog distribution function to reduce the excess numerical diffusion in the first order relaxation scheme is novel. Moreover, our scheme avoids intricate and time consuming solving of Riemann problems and complicated flux splittings. In [38] Raghurama Rao and Subba Rao has introduced a relaxation scheme based on characteristics and interpolation which does not require the discretization of any derivatives. Our scheme also possess this new feature and which makes our approach different from the traditional finite difference, finite volume and finite element methods.

The organisation of this paper is as follows. In section 2 we introduce a relaxation system for Euler equations in the form of a discrete velocity Boltzmann equation. In section 3 we derive a first order accurate, unconditionally stable relaxation scheme which is continuous in space and discrete 
in time. In order to get a fully discrete scheme, we use a simple interpolation strategy. We prove the positivity preserving property and entropy stability of the first order scheme. In section 4 we present an extension of the first order scheme to second order with the aid of an anti-diffusive Chapman-Enskog distribution function. The results of numerical experiments on some benchmark problems are reported in section 5. Finally, we conclude the paper with some remarks in section 6 .

\section{Relaxation System for Euler Equations}

In this section we introduce a relaxation system for the one-dimensional Euler equations of an inviscid compressible fluid. However, the generalisation of this idea to any complex system of conservation laws is straight forward. The Euler equations forms a nonlinear hyperbolic system of conservation laws which represents the fundamental conservation principles of mass, momentum and energy. The system of equations reads

$$
\frac{\partial w}{\partial t}+\frac{\partial g(w)}{\partial x}=0
$$

with the vector valued conserved variable $w$ and the flux $g(w)$ given as

$$
w=\left(\begin{array}{c}
\rho \\
\rho u \\
E
\end{array}\right), g(w)=\left(\begin{array}{c}
\rho u \\
\rho u^{2}+p \\
(E+p) u
\end{array}\right) .
$$

Here, $\rho, \rho u$ and $E$ respectively denote the densities of mass, momentum and energy and $p$ is the pressure. In order to close the system (2.1), we assume the equation of state of a polytropic ideal gas so that $p$ is related to the other state variables as $p=(\gamma-1)\left(E-\rho u^{2} / 2\right)$, where $\gamma$ is the ratio of specific heats. The set of values of the state variable $w$ forms the admissible set

$$
\mathcal{W}=\left\{(\rho, \rho u, E)^{t}: \rho>0, u \in \mathbb{R}, E-\frac{\rho u^{2}}{2}>0\right\},
$$

which is an open convex subset of $\mathbb{R}^{3}$. It is to be noted that the differential equations (2.1) hold only at regular points and for weak solutions containing singular surfaces (2.1) is to be replaced by the jump condition

$$
-s \llbracket w \rrbracket+\llbracket g(w) \rrbracket=0,
$$

where $s$ is the normal speed of the singular surface. However, weak solutions of conservation laws are not unique. The physically relevant unique weak solution can be obtained using the entropy condition, which is a reminiscent of the second law of thermodynamics. For example, for the Euler equations (2.1) a strictly convex entropy function $h$ and the associated entropy flux $\varphi$ can written as a function of the density $\rho$ and pressure $p$ as

$$
h(\rho, p)=c_{v} \log \left(\frac{p}{\rho^{\gamma}}\right), \varphi(\rho, p)=\rho h(\rho, p) u .
$$

The relaxation problems occur quite often in many physical problems, for example in nonequilibrium and extended thermodynamics $[8,25]$, kinetic theory $[6,7]$ and nonlinear waves [45]. A relaxation phenomenon arises when the equilibrium state of a physical system is perturbed. One of most common occurrence of relaxation processes is in rarefied gas dynamics which represented by the well known Boltzmann equation. The Boltzmann equation for a monatomic perfect gas in one space dimension is given by

$$
\frac{\partial f}{\partial t}+\xi \frac{\partial f}{\partial x}=\frac{1}{\epsilon} Q(f)
$$


where $f(x, t, \xi) \geq 0$ is the particle density function. The physical conserved variables, viz., mass, momentum and energy, are obtained from the moment relations

$$
w(x, t)=\int_{\mathbb{R}} \Psi(\xi) f(x, t, \xi) \mathrm{d} \xi,
$$

where

$$
\Psi(\xi)=\left(\begin{array}{c}
1 \\
\xi \\
\frac{1}{2} \xi^{2}
\end{array}\right)
$$

The Boltzmann collision operator $Q$ consists of a very complex integral term. An interesting property of this collision operator $Q$ is that $Q(f)=0$ if and only if $f$ is a Maxwellian, i.e.,

$$
f(x, t, \xi)=M(w, \xi):=\frac{\rho}{(2 \pi \vartheta)^{1 / 2}} e^{-\frac{|\xi-u|^{2}}{2 \vartheta}},
$$

where $\vartheta=R T, R$ being the gas constant.

In their work [3], Bhatnagar, Gross and Krook introduced a simple model for $Q$ based on the relaxation process of a swarm of molecules towards an equilibrium state. With this hierarchy, the so called BGK model, the Boltzmann equation (2.6) reads

$$
\frac{\partial f}{\partial t}+\xi \frac{\partial f}{\partial x}=\frac{1}{\epsilon}\left(M\left(w_{f}, \xi\right)-f\right),
$$

where $\epsilon>0$ is a small parameter known as the relaxation time and $w_{f}$ is defined by

$$
w_{f}=\int_{\mathbb{R}} \Psi(\xi) f(x, t, \xi) \mathrm{d} \xi .
$$

Here we wish to put a subscript $f$ on $w$, just to emphasise that in the construction of $M$ in (2.10), $w_{f}$ is the macroscopic conserved variable obtained from $f$. The BGK Boltzmann equation (2.10) facilitated the development of kinetic schemes, which have been very successful in the numerical modelling of many initial and boundary value problems in fluid dynamics. The kinetic schemes to solve the compressible Euler equations have been mainly developed in the works of Sanders and Prendergast [39], Pullin [34], Deshpande [11, 12, 13], Perthame [31, 32], Raghurama Rao and Deshpande [37] and $\mathrm{Xu}[46,47]$. These schemes are based on the fact that the Euler equations (2.1) are the first moments of the Boltzmann equation (2.6) when the distribution function is the Maxwellian $(2.9)$, see $[6,7]$. The kinetic schemes admit many fascinating features: robustness, upwind bias, preserving the positivity of mass density and pressure, entropy stability, etc.

As a generalisation of kinetic BGK models, in [4] Bouchut has introduced a general framework for constructing a BGK model for any system of conservation laws endowed with a convex entropy. A striking property of this formulation is that the constructed BGK model possesses a large family of kinetic entropies. There exists an analogue of the classical Boltzmann $H$-theorem [6, 7], the exploitation of which yields the entropy inequality in the hydrodynamic limit, see [4] for more details. In this work we use the discrete velocity relaxation model introduced by Aregba-Driollet and Natalini [1], Bouchut [4]. The BGK equation reads

$$
\frac{\partial f_{k}}{\partial t}+a(k) \frac{\partial f_{k}}{\partial x}=\frac{1}{\epsilon}\left(M_{k}\left(w_{f}\right)-f_{k}\right)
$$

for $k \in\{1,2, \ldots, N\}$. Here $f_{k}=f_{k}(x, t) \in \mathbb{R}^{3}$ is unknown, $a(k) \in \mathbb{R}$ is a constant, $w_{f}=\sum_{k=1}^{N} f_{k}$ and the so called Maxwellians $M_{k}: \mathcal{W} \rightarrow \mathbb{R}^{3}$ satisfy the consistency conditions

$$
\sum_{k=1}^{N} M_{k}(w)=w, \sum_{k=1}^{N} a(k) M_{k}(w)=g(w), w \in \mathcal{W} .
$$


The conditions (2.13) are the necessary conditions for the BGK model (2.12) to converge to the Euler equations $(2.1)$ in the limit $\epsilon \rightarrow 0$, see $[1,26]$ for more details. We note that the BGK model (2.12) is completely determined once the discrete velocities $a(k)$ and the Maxwellians $M_{k}$ are obtained. The choices of $a(k)$ and $M_{k}$ are to be done according to some suitable stability conditions. It is well known that even for general relaxation models $[9,24,26,40]$ an approximation of the type (2.12) has to obey some stability criterion so as to possess the correct hydrodynamic limit. In the case of $2 \times 2$ relaxation systems it is the well known sub-characteristic condition [9, 24], see [5] for a survey of different stability conditions for relaxation problems. We use the entropy extension condition of Bouchut [4] so that the BGK model (2.12) is compatible with the entropies of (2.1). The main result of [4] for the discrete velocity BGK models of the type (2.12) states: under the necessary and sufficient condition

$$
\sigma\left(M_{k}^{\prime}(w)\right) \subset[0, \infty) \forall k
$$

corresponding to any entropy $h(w)$ of $(2.1)$, there exist a kinetic entropy $H_{k}\left(f_{k}\right)$ of $(2.12)$ such that

(i) $H_{k}$ is a convex function,

(ii) $\sum_{k=1}^{N} H_{k}\left(M_{k}(w)\right)=h(w)$,

(iii) $\sum_{k=1}^{N} H_{k}\left(M_{k}\left(w_{f}\right)\right) \leq \sum_{k=1}^{N} H_{k}\left(f_{k}\right)$.

We now proceed to give the explicit expressions for the discrete velocities $a(k)$ and the Maxwellians $M_{k}$ in accordance with the stability requirement (2.14). Firstly, we choose $N=2$ and take

$$
a(1)=-\lambda, a(2)=\lambda,
$$

where $\lambda$ is a parameter to be determined. In order to satisfy (2.14), we choose $M_{k}$ to be a linear combination of $w$ and $g(w)$, i.e.,

$$
M_{k}(w)=\alpha_{k} w+\beta_{k} g(w)
$$

Using (2.15), the relations (2.13) immediately give the expressions

$$
\begin{aligned}
& M_{1}(w)=\frac{1}{2} w-\frac{1}{2 \lambda} g(w), \\
& M_{2}(w)=\frac{1}{2} w+\frac{1}{2 \lambda} g(w) .
\end{aligned}
$$

Note that an expression for the parameter $\lambda$ remains to be determined. The eigenvalues of the Jacobian $A(w)=\partial g(w) / \partial w$ are $u-a, u, u+a$, where $a=\sqrt{\gamma p / \rho}$ is the sound speed. Evaluating the expression on the right hand side of (2.14) yields the expression for $\lambda$ as

$$
\lambda=\sup (|u|+a) \text {. }
$$

Remark 2.1. If we assume that the functions $\left\{M_{1}, M_{2}\right\}$ are continuously differentiable, then $\left\{M_{1}, M_{2}\right\}$ gives a wave-model [28] for the system (2.1) with $\{a(1), a(2)\}$ as the corresponding advection velocities. The relation (2.13) precisely shows that this wave-model is consistent with both the state-vector $w$ and the flux-vector $g(w)$. In other words, we can decompose the state-vector $w$ into two waves $M_{1}(w)$ and $M_{2}(w)$ which advects with velocities $a(1)$ and $a(2)$ respectively.

\section{Kinetic Relaxation Scheme}

In this section we derive a first order accurate, unconditionally stable discrete kinetic scheme for the Euler equations (2.1) using the discrete velocity Boltzmann equation (2.12). For an analogous 
formulation in the context of classical kinetic schemes, see [12, 22]. Firstly, we start with a bounded, integrable initial data for the macroscopic variables, i.e.,

$$
\rho(x, 0)=\rho_{0}(x)>0, u(x, 0)=u_{0}(x), p(x, 0)=p_{0}(x)>0 .
$$

Let us denote the solution at time $t=t^{n}$ by $w^{n}(x)$, i.e., $w^{n}(x) \sim w\left(x, t^{n}\right)$. Using the values of $w^{n}(x)$ we obtain $\lambda$ from (2.19) and form the Maxwellian densities $M_{k}\left(w^{n}(x)\right)$. In other words, we assume that the distribution function relaxes instantaneously to the Maxwellian at time $t=t^{n}$, i.e., $f_{k}\left(x, t^{n}\right)=M_{k}\left(w^{n}(x)\right)$. This process has been referred to as collision phase in the literature of kinetic schemes $[12,15]$. In the next stage we solve the initial value problem for the collision free Boltzmann equation

$$
\begin{aligned}
\frac{\partial f_{k}}{\partial t}+a(k) \frac{\partial f_{k}}{\partial x} & =0, \\
f_{k}\left(x, t^{n}\right) & =M_{k}\left(w^{n}(x)\right) .
\end{aligned}
$$

The initial value problem (3.2)-(3.3) can be solved exactly to yield the solution

$$
\begin{aligned}
f_{k}\left(x, t^{n}+\Delta t\right) & =f_{k}\left(x-a(k) \Delta t, t^{n}\right) \\
& =M_{k}\left(w^{n}(x-a(k) \Delta t)\right) .
\end{aligned}
$$

This leads to an iterative scheme for the macroscopic conserved variable $w$, defined by

$$
w^{n+1}(x)=\sum_{k=1}^{2} M_{k}\left(w^{n}(x-a(k) \Delta t)\right) .
$$

Thus, our numerical scheme consists of two phases: a collision phase and a convection phase. In the collision phase the distribution function $f_{k}$ relaxes instantaneously to the equilibrium distribution $M_{k}(w)$. It tantamount to performing particle collisions instantaneously to make the transition from a non-equilibrium state to an equilibrium state. On the other hand, the convection phase drives the system away from the equilibrium state, i.e., $f_{k}$ becomes more and more different from the equilibrium $M_{k}$. Therefore, our numerical scheme (3.5), derived in the spirit of kinetic schemes is termed as kinetic relaxation scheme (KRS), see [35] for some analogous formulations of relaxation schemes. It is interesting to note that this KRS (3.5) is discrete in time, but continuous in space. Further, it is unconditionally stable, i.e., it does not require any restriction on the time-step $\Delta t$.

Remark 3.1. It has been proved by Jin [19] that a simple splitting strategy of the type we employed here reduces the resulting scheme to formally first order accurate in time. Therefore, the scheme (3.5) is only first order accurate in time.

The rest of this section is devoted to analysis of the scheme (3.5). Firstly, we derive a fully discrete and conditionally stable scheme from (3.5). Let us introduce a mesh, which for simplicity is assumed to be uniform with mesh size $\Delta x$. We denote by $w_{j}^{n}$, the point value of $w$ at $x=x_{j}$ at time $t=t^{n}$. It has to be noted that the formula involves the values of $M_{k}(w)$ at non-mesh points, see Figure 1.

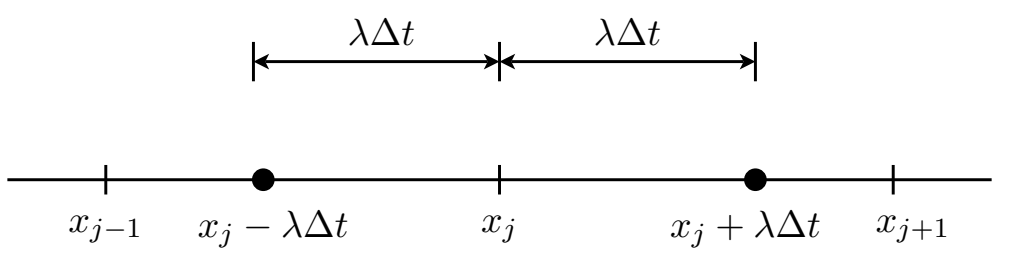

Figure 1. Computational stencil used in the interpolation scheme. 
As in the classical kinetic scheme $[12,22]$ we use an interpolation scheme to evaluate the term on the right hand side of (3.5). Since the set $\left\{M_{1}, M_{2}\right\}$ represents two waves travelling to the left and right respectively, we introduce an upwind bias in interpolating via

$$
M_{1}\left(w^{n}\left(x_{j}+\lambda \Delta t\right)\right)=M_{1 j}^{n}+\frac{\lambda \Delta t}{\Delta x}\left(M_{1 j+1}^{n}-M_{1 j}^{n}\right),
$$

where $M_{1 j}^{n}$ is a shortcut for $M_{1}\left(w^{n}\left(x_{j}\right)\right)$. In an analogous manner we derive

$$
M_{2}\left(w^{n}\left(x_{j}-\lambda \Delta t\right)\right)=M_{2 j}^{n}-\frac{\lambda \Delta t}{\Delta x}\left(M_{2 j}^{n}-M_{2 j-1}^{n}\right) .
$$

Introducing (3.6)-(3.7) in (3.5) finally yields the fully discrete scheme

$$
w_{j}^{n+1}=M_{1 j}^{n}+\frac{\lambda \Delta t}{\Delta x}\left(M_{1 j+1}^{n}-M_{1 j}^{n}\right)+M_{2 j}^{n}-\frac{\lambda \Delta t}{\Delta x}\left(M_{2 j}^{n}-M_{2 j-1}^{n}\right) .
$$

3.1. Conservation Property of the Scheme. It is not however, apparent that the difference scheme (3.8) is conservative, i.e., it possesses discrete versions of the fundamental conservation laws of mass, momentum and energy. We now prove that (3.8) can be recast into a conservative scheme.

Proposition 3.2. The numerical scheme (3.8) can be written as a conservative difference scheme

$$
\frac{w_{j}^{n+1}-w_{j}^{n}}{\Delta t}+\frac{\mathcal{G}_{j+\frac{1}{2}}-\mathcal{G}_{j-\frac{1}{2}}}{\Delta x}=0,
$$

where the numerical flux $\mathcal{G}_{j+\frac{1}{2}}$ is defined by

$$
\mathcal{G}_{j+\frac{1}{2}}\left(w_{j+1}^{n}, w_{j}^{n}\right)=\frac{1}{2}\left(g\left(w_{j+1}^{n}\right)+g\left(w_{j}^{n}\right)\right)-\frac{\lambda}{2}\left(w_{j+1}^{n}-w_{j}^{n}\right) .
$$

Proof. We use the expressions (2.17)-(2.18) for $M_{1}, M_{2}$ and the consistency conditions (2.13) in (3.8). Rearranging the terms yields (3.9).

Remark 3.3. It has to be noted that the numerical flux $\mathcal{G}_{j+1 / 2}$ in (3.9) contains the parameter $\lambda$ explicitly. From (2.19) we infer that $\lambda$ depends on the values of $w$ in the whole domain. Therefore, the conservative equation (3.9) is a non-local relation.

Remark 3.4. As mentioned earlier in Remark 2.1, the Maxwellians $\left\{M_{1}, M_{2}\right\}$ gives a flux consistent wave decomposition, i.e., the flux vector $g(w)$ can be split as

$$
\begin{aligned}
g(w) & =-\lambda M_{1}(w)+\lambda M_{2}(w) \\
& =g^{+}(w)+g^{-}(w),
\end{aligned}
$$

where $g^{+}(w)=\lambda M_{2}(w)$ and $g^{-}(w)=-\lambda M_{1}(w)$. As a result of (3.11), the numerical flux $\mathcal{G}_{j+\frac{1}{2}}$ can be written as the sum of split fluxes

$$
\mathcal{G}_{j+\frac{1}{2}}\left(w_{j+1}^{n}, w_{j}^{n}\right)=g^{+}\left(w_{j}^{n}\right)+g^{-}\left(w_{j+1}^{n}\right) .
$$

As in the classical kinetic schemes, the flux decomposition (3.12) is the result of treating the continuum as an ensemble of particles. The movement of particles to the left and right naturally gives a splitting of the fluxes of mass, momentum and energy into negative and positive parts. This is the fundamental idea behind the kinetic flux-vector splitting (KFVS) scheme introduced by Pullin [34], Deshpande [13]. Therefore, our scheme (3.9) also shares the spirit of KFVS scheme. 
3.2. Positivity Preserving Property. One of the most important characteristics of kinetic schemes is their positivity preserving property under a suitable CFL stability condition $[15,31,32]$. In what follows, we prove that the discrete kinetic scheme (3.9) also preserves the positivity of the mass density and pressure. This is a very desirable property, particularly for problems involving nearly vacuum states. It is well known that many of the Riemann solver based schemes do not possess this feature, see [14] for more details. For the kinetic schemes, the positivity preserving property implies the $L^{1}$-stability. We now prove that the discrete kinetic scheme also admits the same feature.

Theorem 3.5. Under the CFL condition

$$
\lambda \frac{\Delta t}{\Delta x} \leq 1
$$

and $\lambda$ chosen according to the stability condition (2.14), the discrete kinetic scheme (3.9) preserves the positivity of mass density and pressure, i.e.,

$$
\rho_{j}^{n} \geq 0, p_{j}^{n} \geq 0, \forall j \Rightarrow \rho_{j}^{n+1} \geq 0, p_{j}^{n+1} \geq 0, \forall j
$$

Further, the scheme is $L^{1}$-stable.

Remark 3.6. Under some technical assumptions on the Maxwellian densities, it has been proved in [4] that the BGK model (2.12) preserves the positivity of mass density and pressure. We now prove that our discretization also maintains the same characteristic, i.e., we establish the positivity property for the fully discrete scheme (3.9). In fact, the positivity of the mass density can be readily inferred as follows. Note that the first components of $M_{1}$ and $M_{2}$ are given by

$$
\begin{aligned}
& M_{1}(w)_{1}=\frac{\rho}{2}\left(1-\frac{u}{\lambda}\right), \\
& M_{2}(w)_{1}=\frac{\rho}{2}\left(1+\frac{u}{\lambda}\right) .
\end{aligned}
$$

Using the assumptions of the theorem 3.5 and the stability condition (2.14), the right hand sides of both the expressions in (3.15) are positive. From (3.8) it is now easy to see that under the CFL condition (3.13), the first component of the vector valued expression on the right hand side is positive, i.e., $\rho_{j}^{n+1}$ is positive.

However, in order to give a complete proof we proceed as follows. In [15] Estivales and Villedieu has given a characterisation for a flux-splitting scheme to preserve the positivity of mass density and pressure. Since the scheme (3.9) also admits a flux decomposition (3.12), we make use of the theorem of [15] to establish the result.

Proof of theorem 3.5. Let us assume that $\rho$ and $p$ remain positive at all mesh points at time $t=t^{n}$, i.e.,

$$
\rho_{j}^{n} \geq 0, E_{j}^{n}-\frac{\left(m_{j}^{n}\right)^{2}}{2 \rho_{j}^{n}} \geq 0, \forall j,
$$

where $m_{j}^{n}=\rho_{j}^{n} u_{j}^{n}$. In other words, we assume that $w_{j}^{n} \in \mathcal{W}$. Therefore, according to the theorem 2.1 of [15], in order to prove the positivity preserving property, it is sufficient to show that $g^{+}\left(w_{j}^{n}\right) \in \mathcal{W}$. 
We denote the components of $g^{+}\left(w_{j}^{n}\right)$ by $\mathfrak{r}_{j}^{n}, \mathfrak{m}_{j}^{n}$ and $\mathfrak{E}_{j}^{n}$ respectively, i.e.,

$$
\begin{aligned}
\mathfrak{r}_{j}^{n} & =\frac{1}{2}\left(\lambda \rho_{j}^{n}+m_{j}^{n}\right), \\
\mathfrak{m}_{j}^{n} & =\frac{1}{2}\left(\lambda m_{j}^{n}+\left(\frac{\left(m_{j}^{n}\right)^{2}}{\rho_{j}^{n}}+p_{j}^{n}\right)\right), \\
\mathfrak{E}_{j}^{n} & =\frac{1}{2}\left(\lambda E_{j}^{n}+\left(E_{j}^{n}+p_{j}^{n}\right) \frac{m_{j}^{n}}{\rho_{j}^{n}}\right) .
\end{aligned}
$$

We need to show that $\mathfrak{r}_{j}^{n}>0$ and $\mathfrak{E}_{j}^{n}-\left(\mathfrak{m}_{j}^{n}\right)^{2} /\left(2 \mathfrak{r}_{j}^{n}\right)>0$. Clearly,

$$
\mathfrak{r}_{j}^{n}=\frac{1}{2} \rho_{j}^{n}\left(\lambda+u_{j}^{n}\right)>0 .
$$

Therefore, the density component of $g^{+}\left(w_{j}^{n}\right)$ is positive. We now prove the same for the pressure like term. Now,

$$
\mathfrak{E}_{j}^{n}-\left(\mathfrak{m}_{j}^{n}\right)^{2} /\left(2 \mathfrak{r}_{j}^{n}\right)=\frac{2 \mathfrak{E}_{j}^{n} \mathfrak{r}_{j}^{n}-\left(\mathfrak{m}_{j}^{n}\right)^{2}}{2 \mathfrak{r}_{j}^{n}}
$$

Using the expressions from (3.17)-(3.19) yields

$$
\begin{aligned}
2 \mathfrak{E}_{j}^{n} \mathfrak{r}_{j}^{n}-\left(\mathfrak{m}_{j}^{n}\right)^{2} & =\frac{1}{2 \rho_{j}^{n}}\left(E_{j}^{n}-\frac{\left(m_{j}^{n}\right)^{2}}{2 \rho_{j}^{n}}\right)\left(\lambda \rho_{j}^{n}+m_{j}^{n}\right)^{2}-\frac{\left(p_{j}^{n}\right)^{2}}{4} \\
& =\frac{\rho_{j}^{n} p_{j}^{n}}{2(\gamma-1)}\left(\lambda+u_{j}^{n}\right)^{2}-\frac{\left(p_{j}^{n}\right)^{2}}{4} \\
& =\frac{\rho_{j}^{n} p_{j}^{n}}{2}\left(\frac{\left(\lambda+u_{j}^{n}\right)^{2}}{\gamma-1}-\frac{\left(a_{j}^{n}\right)^{2}}{2 \gamma}\right) \\
& >0 .
\end{aligned}
$$

Note that here we have used $a^{2}=\gamma p / \rho$. Thus, the proof of positivity property is completed. We next consider the $L^{1}$-stability. Since the quantities $\rho$ and $E$ remain positive and the scheme is conservative, we have

$$
\begin{aligned}
\left\|\rho^{n+1}\right\|_{L^{1}} & =\sum_{j \in \mathbb{Z}} \rho_{j}^{n+1} \\
& =\sum_{j \in \mathbb{Z}} \rho_{j}^{n} \\
& =\left\|\rho^{n}\right\|_{L^{1}} .
\end{aligned}
$$

In an analogous manner we can show

$$
\left\|E^{n+1}\right\|_{L^{1}}=\left\|E^{n}\right\|_{L^{1}}
$$


Now

$$
\begin{aligned}
\left\|\rho^{n+1} u^{n+1}\right\|_{L^{1}} & =\sum_{j \in \mathbb{Z}} \rho_{j}^{n+1}|u|_{j}^{n+1} \\
& =\sum_{j \in \mathbb{Z}}\left(\rho_{j}^{n+1}\right)^{1 / 2}\left(\rho_{j}^{n+1}\left(u_{j}^{n+1}\right)^{2}\right)^{1 / 2} \\
& \leq\left(\sum_{j \in \mathbb{Z}} \rho_{j}^{n+1}\right)^{1 / 2}\left(\sum_{j \in \mathbb{Z}} \rho_{j}^{n+1}\left(u_{j}^{n+1}\right)^{2}\right)^{1 / 2} \\
& \leq 2\left\|\rho^{n+1}\right\|_{L^{1}}^{1 / 2}\left(\sum_{j \in \mathbb{Z}} E_{j}^{n+1}\right)^{1 / 2} \\
& =2\left\|\rho^{n+1}\right\|_{L^{1}}^{1 / 2}\left\|E^{n+1}\right\|_{L^{1}}^{1 / 2} \\
& =2\left\|\rho^{n}\right\|_{L^{1}}^{1 / 2}\left\|E^{n}\right\|_{L^{1}}^{1 / 2} \\
& \leq\left\|\rho^{n}\right\|_{L^{1}}+\left\|E^{n}\right\|_{L^{1}} .
\end{aligned}
$$

Hence, the proof of $L^{1}$ stability is complete.

3.3. Entropy stability of the Scheme. Yet another important feature of kinetic schemes is their entropy stability property, which is a consequence of the Boltzmann $H$-theorem. Our next aim is to establish the same property for our scheme (3.9). In the next theorem, we prove that there exists a discrete entropy inequality for the scheme (3.9).

Theorem 3.7. Under the CFL condition

$$
\lambda \frac{\Delta t}{\Delta x} \leq 1
$$

and $\lambda$ chosen according to the stability condition (2.14), the discrete kinetic scheme (3.9) is entropy stable, i.e., it satisfies the discrete entropy inequality

$$
\frac{h\left(w_{j}^{n+1}\right)-h\left(w_{j}^{n}\right)}{\Delta t}-\frac{\Phi_{j+\frac{1}{2}}-\Phi_{j-\frac{1}{2}}}{\Delta x} \leq 0,
$$

where the entropy flux $\Phi_{j+\frac{1}{2}}$ is given by

$$
\Phi_{j+\frac{1}{2}}\left(w_{j+1}^{n}, w_{j}^{n}\right)=\lambda H_{2}\left(M_{2}\left(w_{j}^{n}\right)\right)-\lambda H_{1}\left(M_{1}\left(w_{j+1}^{n}\right)\right) .
$$

Proof.

$$
\begin{aligned}
f_{1 j}^{n+1} & =f_{1}\left(x_{j}, t^{n}+\Delta t\right) \\
& =f_{1}\left(x_{j}-a(1) \Delta t, t^{n}\right) \\
& =M_{1}\left(w^{n}\left(x_{j}+\lambda \Delta t\right)\right) \\
& =\frac{\lambda \Delta t}{\Delta x} M_{1 j+1}^{n}+\left(1-\frac{\lambda \Delta t}{\Delta x}\right) M_{1 j}^{n} .
\end{aligned}
$$

Since $H_{1}$ is a convex function, an application of Jensen's inequality yields

$$
H_{1}\left(f_{1 j}^{n+1}\right) \leq \frac{\lambda \Delta t}{\Delta x} H_{1}\left(M_{1 j+1}^{n}\right)+\left(1-\frac{\lambda \Delta t}{\Delta x}\right) H_{1}\left(M_{1 j}^{n}\right) .
$$


In an analogous manner we can derive

$$
H_{2}\left(f_{2}^{n+1}\right) \leq \frac{\lambda \Delta t}{\Delta x} H_{2}\left(M_{2 j-1}^{n}\right)+\left(1-\frac{\lambda \Delta t}{\Delta x}\right) H_{2}\left(M_{2 j}^{n}\right) .
$$

We note that

$$
\begin{aligned}
h\left(w_{j}^{n+1}\right) & =H_{1}\left(M_{1}\left(w_{j}^{n+1}\right)\right)+H_{2}\left(M_{2}\left(w_{j}^{n+1}\right)\right) \\
& \leq H_{1}\left(f_{1 j}^{n+1}\right)+H_{2}\left(f_{2 j}{ }^{n+1}\right) .
\end{aligned}
$$

We now add (3.27) and (3.28) and rearrange the terms, which gives the result.

Remark 3.8. Like the numerical flux $\mathcal{G}_{j+\frac{1}{2}}$ in (3.9), the entropy flux $\Phi_{j+\frac{1}{2}}$ can also be decomposed into a positive and negative part

$$
\Phi_{j+\frac{1}{2}}\left(w_{j+1}^{n}, w_{j}^{n}\right)=\varphi^{+}\left(w_{j}^{n}\right)+\varphi^{-}\left(w_{j+1}^{n}\right),
$$

where the split fluxes are given by

$$
\varphi^{+}(w)=\lambda H_{2}\left(M_{2}(w)\right), \varphi^{-}(w)=-\lambda H_{1}\left(M_{1}(w)\right) .
$$

\section{Second Order Accurate Kinetic Relaxation Scheme}

In this section we extend our kinetic relaxation scheme (3.5) to second order. We follow the approach of Deshpande [12] which he used to obtain a second order kinetic scheme for the compressible Euler equations of gas dynamics. The first order fully discrete scheme (3.8) has many desirable properties, such as it is conservative, positivity preserving and entropy stable. However, it suffers from a large amount of numerical dissipation. It was remarked in $[12,22]$ that for first order kinetic schemes, the numerical dissipation is proportional to the time-step $\Delta t$. We shall see later in this section that it is true also for the discrete kinetic scheme (3.8). Following Deshpande [12], we employ a Chapman-Enskog type expansion to derive a higher order numerical dissipation. The resulting scheme will then be second order accurate.

There are two steps in deriving a second order scheme. In the first step we proceed to achieve second order accuracy in time. For this we employ the Chapman-Enskog type procedure, which leads to an anti-diffusive flux correction to gain second order time accuracy. The second step is to achieve second order accuracy in space, which consists of using a second order interpolation strategy.

4.1. Second Order Accuracy in Time. Expanding the exact solution $w(x, t)$ in Taylor series to second order accuracy yields

$$
w\left(x, t^{n}+\Delta t\right)=w\left(x, t^{n}\right)+\Delta t \frac{\partial w}{\partial t}\left(x, t^{n}\right)+\frac{\Delta t^{2}}{2} \frac{\partial^{2} w}{\partial t^{2}}\left(x, t^{n}\right)+\mathcal{O}\left(\Delta t^{3}\right) .
$$

Note that the Taylor expansion (4.1) contains first and second time derivatives of $w$. We make use of the conservation law (2.1) to replace this time derivatives by space derivatives to obtain

$$
w\left(x, t^{n}+\Delta t\right)=w\left(x, t^{n}\right)-\Delta t \frac{\partial g(w)}{\partial x}\left(x, t^{n}\right)+\frac{\Delta t^{2}}{2} \frac{\partial}{\partial x}\left(A(w)^{2} \frac{\partial w}{\partial x}\right)\left(x, t^{n}\right)+\mathcal{O}\left(\Delta t^{3}\right) .
$$

Our aim is to compare (4.2) with a corresponding second order Taylor expansion of the right hand side of (3.5). This comparison will give us the missing terms in the first order kinetic relaxation scheme, the so called anti-diffusive terms. The addition of these terms to the first order scheme 
enables us to gain second order accuracy in time. In order to proceed, we expand the first term on the right hand side of (3.5) to second order accuracy, resulting in

$M_{1}\left(w^{n}(x-a(1) \Delta t)\right)=M_{1}\left(w^{n}(x+\lambda \Delta t)\right)$

$$
=M_{1}\left(w^{n}\left(x, t^{n}\right)\right)+\lambda \Delta t \frac{\partial M_{1}}{\partial x}\left(\left(w^{n}\left(x, t^{n}\right)\right)+\frac{\lambda^{2} \Delta t^{2}}{2} \frac{\partial^{2} M_{1}}{\partial x^{2}}\left(w^{n}\left(x, t^{n}\right)\right)+\mathcal{O}\left(\Delta t^{3}\right) .\right.
$$

In an analogous way we obtain

$M_{2}\left(w^{n}(x-a(2) \Delta t)\right)=M_{2}\left(w^{n}\left(x, t^{n}\right)\right)-\lambda \Delta t \frac{\partial M_{2}}{\partial x}\left(w^{n}\left(x, t^{n}\right)\right)+\frac{\lambda^{2} \Delta t^{2}}{2} \frac{\partial^{2} M_{2}}{\partial x^{2}}\left(w^{n}\left(x, t^{n}\right)\right)+\mathcal{O}\left(\Delta t^{3}\right)$.

Adding (4.3) and (4.4), making use of (3.5) and the moment relations (2.13) yields

$$
w\left(x, t^{n}+\Delta t\right)=w\left(x, t^{n}\right)-\Delta t \frac{\partial g(w)}{\partial x}\left(x, t^{n}\right)+\frac{\lambda^{2} \Delta t^{2}}{2} \frac{\partial^{2} w}{\partial x^{2}}\left(x, t^{n}\right)+\mathcal{O}\left(\Delta t^{3}\right) .
$$

Notice that (4.5) is the modified partial differential equation (MPDE) for the scheme (3.5). It can be observed that the diffusion term is of $\mathcal{O}(\Delta t)$ as in the classical kinetic schemes. We can now rewrite the second order Taylor expansion (4.2) by adding and subtracting the $\mathcal{O}\left(\Delta t^{2}\right)$ term appearing in (4.5) to get

$$
\begin{aligned}
w\left(x, t^{n}+\Delta t\right) & =w\left(x, t^{n}\right)-\Delta t \frac{\partial g(w)}{\partial x}\left(x, t^{n}\right)+\frac{\Delta t^{2}}{2} \frac{\partial}{\partial x}\left(\lambda^{2} \frac{\partial w}{\partial x}\right) \\
-\frac{\Delta t^{2}}{2} \frac{\partial}{\partial x}\left(\left(\lambda^{2} I-A(w)^{2}\right) \frac{\partial w}{\partial x}\right)+\mathcal{O}\left(\Delta t^{3}\right) & \\
= & \sum_{k=1}^{2} M_{k}\left(w^{n}(x-a(k) \Delta t)\right)+\Delta t \frac{\partial \mathcal{D}}{\partial x}+\mathcal{O}\left(\Delta t^{3}\right),
\end{aligned}
$$

where we define

$$
\mathcal{D}=-\frac{\Delta t}{2} \mathcal{B} \frac{\partial w}{\partial x}, \mathcal{B}=\left(\lambda^{2} I-A(w)^{2}\right) .
$$

Here $I$ denotes the $3 \times 3$ identity matrix. It has been proved in [4] that under the stability condition (2.14), the matrix $\mathcal{B}$ has nonnegative real eigenvalues. Therefore, $\mathcal{D}$ behaves like a viscous stress term. This new stress term $\mathcal{D}$ is analogous to the heat flux vector and viscous stress obtained by Deshpande [12] for the compressible Euler equations using similar arguments. The gradient of $\mathcal{D}$, i.e., $\partial \mathcal{D} / \partial x$ will act as dissipative flux. At this point, it is very important to note that sign of $\mathcal{D}$ in (4.7) is negative. As a result, the term $\partial \mathcal{D} / \partial x$ in (4.6) is a negative diffusive flux. In other words, it is an anti-diffusive flux. Note that the first term in (4.6) is coming from the first order scheme (3.5). Hence, in order to achieve second order time accuracy for the discrete kinetic scheme (3.8) we need to consider not only the upwind relaxation term but also the anti-diffusive term. Further, the antidiffusive term reduces the excess amount of numerical diffusion present in the upwind relaxation scheme (3.8).

Notice that in the second order scheme (4.6) we have incorporated a diffusive flux term. However, it is a characteristic of the Maxwellian equilibrium distributions of the type $M_{k}(w)$ to give an inviscid system of conservation laws in the hydrodynamic limit, see $[4,9]$. Therefore, in order to get a dissipative flux like term $\partial \mathcal{D} / \partial x$ we need to change the Maxwellian distribution to a ChapmanEnskog distribution. The latter is always associated with the Navier-Stokes equation and hence it can give rise to nonzero viscous terms. Moreover, the method of replacing the time derivatives by space derivatives we performed to get (4.2) is a characteristic of the Chapman-Enskog procedure. We now proceed to derive a Chapman-Enskog distribution and show that the second order accurate 
scheme (4.6) can be recast in the form (3.5) using the Chapman-Enskog distribution instead of the Maxwellians $M_{k}(w)$.

From (2.12) we infer that $M_{k}\left(w_{f}\right)-f_{k}=\mathcal{O}(\epsilon)$ and as a result

$$
\begin{aligned}
f_{k} & =M_{k}\left(w_{f}\right)-\epsilon\left\{\frac{\partial f_{k}}{\partial t}+a(k) \frac{\partial f_{k}}{\partial x}\right\} \\
& =M_{k}\left(w_{f}\right)-\epsilon\left\{\frac{\partial M_{k}\left(w_{f}\right)}{\partial t}+a(k) \frac{\partial M_{k}\left(w_{f}\right)}{\partial x}\right\}+\mathcal{O}\left(\epsilon^{2}\right) .
\end{aligned}
$$

Note that the right hand side of (4.8) is a perturbation of the Maxwellian $M_{k}$. Motivated by this, our new ansatz, viz. the Chapman-Enskog distribution function $\tilde{M}_{k}$ is defined by

$$
\tilde{M}_{k}(w)=M_{k}(w)-\tau\left\{\frac{\partial M_{k}(w)}{\partial t}+a(k) \frac{\partial M_{k}(w)}{\partial x}\right\},
$$

where $\tau$ is a parameter to be determined. Analogous to (2.13), the Chapman-Enskog distribution function $\tilde{M}_{k}(w)$ is required to satisfy the moment relations

$$
\sum_{k=1}^{2} \tilde{M}_{k}(w)=w, \sum_{k=1}^{2} a(k) \tilde{M}_{k}(w)=g(w)+\mathcal{D} .
$$

Note that the first relation in (4.10) is the conservation property. The second relation precisely states that unlike the Maxwellian, the Chapman-Enskog distribution should give a nonzero viscous flux in addition to the inviscid flux. We now obtain the precise form $\tilde{M}_{k}(w)$ by evaluating the expressions in curly brackets on the right hand side of (4.9).

$$
\begin{aligned}
\frac{\partial M_{1}(w)}{\partial t} & =\frac{1}{2} \frac{\partial w}{\partial t}-\frac{1}{2 \lambda} \frac{\partial g(w)}{\partial t} \\
& =-\frac{1}{2} A(w) \frac{\partial w}{\partial x}+\frac{1}{2 \lambda} A(w)^{2} \frac{\partial w}{\partial x} .
\end{aligned}
$$

Analogously we obtain

$$
\frac{\partial M_{2}(w)}{\partial t}=-\frac{1}{2} A(w) \frac{\partial w}{\partial x}-\frac{1}{2 \lambda} A(w)^{2} \frac{\partial w}{\partial x} .
$$

Similar calculations shows that

$$
\begin{aligned}
& \frac{\partial M_{1}(w)}{\partial x}=\frac{1}{2} \frac{\partial w}{\partial x}-\frac{1}{2 \lambda} A(w) \frac{\partial w}{\partial x}, \\
& \frac{\partial M_{2}(w)}{\partial x}=\frac{1}{2} \frac{\partial w}{\partial x}+\frac{1}{2 \lambda} A(w) \frac{\partial w}{\partial x} .
\end{aligned}
$$

Thus, we obtain the required expressions for the terms in (4.9)

$$
\begin{aligned}
& \frac{\partial M_{1}(w)}{\partial t}+a(1) \frac{\partial M_{1}(w)}{\partial x}=-\frac{1}{2 \lambda}\left(\lambda^{2} I-A(w)^{2}\right) \frac{\partial w}{\partial x}, \\
& \frac{\partial M_{2}(w)}{\partial t}+a(2) \frac{\partial M_{2}(w)}{\partial x}=\frac{1}{2 \lambda}\left(\lambda^{2} I-A(w)^{2}\right) \frac{\partial w}{\partial x} .
\end{aligned}
$$

Using (4.15)-(4.16) and the expressions for $M_{1}$ and $M_{2}$ in (4.9) yields

$$
\begin{aligned}
& \tilde{M}_{1}(w)=\frac{1}{2} w-\frac{1}{2 \lambda} g(w)-\frac{\tau}{2 \lambda}\left(\lambda^{2} I-A(w)^{2}\right) \frac{\partial w}{\partial x}, \\
& \tilde{M}_{2}(w)=\frac{1}{2} w+\frac{1}{2 \lambda} g(w)+\frac{\tau}{2 \lambda}\left(\lambda^{2} I-A(w)^{2}\right) \frac{\partial w}{\partial x} .
\end{aligned}
$$


The consistency conditions (4.10) immediately gives $\tau=-\Delta t / 2$. Thus, we finally obtain the Chapman-Enskog distribution function

$$
\begin{aligned}
& \tilde{M}_{1}(w)=\frac{1}{2} w-\frac{1}{2 \lambda} g(w)+\frac{\Delta t}{4 \lambda}\left(\lambda^{2} I-A(w)^{2}\right) \frac{\partial w}{\partial x} \\
& \tilde{M}_{2}(w)=\frac{1}{2} w+\frac{1}{2 \lambda} g(w)-\frac{\Delta t}{4 \lambda}\left(\lambda^{2} I-A(w)^{2}\right) \frac{\partial w}{\partial x}
\end{aligned}
$$

It is to be noted that unlike the Maxwellians $M_{k}$, the Chapman-Enskog distribution $\tilde{M}_{k}$ depends also on the derivatives of the conservative variable $w$. In other words, the support of the ChapmanEnskog distribution is larger than the corresponding Maxwellians. The second order accurate kinetic relaxation scheme (4.6) can be recast in an upwind form with the aid of $\tilde{M}_{k}$

$$
w^{n+1}(x)=\sum_{k=1}^{2} \tilde{M}_{k}\left(w^{n}(x-a(k) \Delta t)\right)
$$

4.2. Second Order Accuracy in Space. We now proceed to achieve second order accuracy in space. The equation (4.21) shows that the values of $\tilde{M}_{k}$ are to be evaluated at non-mesh points. This consists of evaluating the two terms on the right hand side of (4.6) to second order accuracy.

In order to compute the first term, i.e., the upwind relaxation term we should employ an interpolation procedure which should be second order accurate. Note that our first order accurate scheme is positivity preserving. Therefore, we must ensure that the second order interpolated values should not give any nonphysical negative density or pressure. As a first step, we use a quadratic interpolation scheme to evaluate the upwind relaxation terms to yield

$$
M_{1}\left(w^{n}\left(x_{j}+\lambda \Delta t\right)\right)=M_{1 j}^{n}+\frac{\eta}{2}\left(M_{1 j+1}^{n}-M_{1 j-1}^{n}\right)+\frac{\eta^{2}}{2}\left(M_{1 j+1}^{n}-2 M_{1 j}^{n}+M_{1 j-1}^{n}\right),
$$

where $\eta=\lambda \Delta t / \Delta x$. An analogous expression for $M_{2}$ is given by

$$
M_{2}\left(w^{n}\left(x_{j}-\lambda \Delta t\right)\right)=M_{2 j}^{n}-\frac{\eta}{2}\left(M_{2 j+1}^{n}-M_{2 j-1}^{n}\right)+\frac{\eta^{2}}{2}\left(M_{2 j+1}^{n}-2 M_{2 j}^{n}+M_{2 j-1}^{n}\right) .
$$

However, as pointed out by Deshpande [12] for classical kinetic schemes, the different components in the vector valued interpolated expressions (4.22)-(4.23) need not be positive even if the corresponding values of $M_{1}$ and $M_{2}$ at the mesh points $j-1, j$ and $j+1$ are positive. This is particularly true in the presence of shocks and high gradients. Adding (4.22) and (4.23) yields

$$
w_{j}^{n+1}=w_{j}^{n}-\frac{\Delta t}{2 \Delta x}\left\{g\left(w_{j+1}^{n}\right)-g\left(w_{j-1}^{n}\right)\right\}+\frac{\lambda^{2} \Delta t^{2}}{\Delta x^{2}}\left(w_{j+1}^{n}-2 w_{j}^{n}+w_{j-1}^{n}\right) .
$$

Thus, we recover a Lax-Wendroff type scheme. It is well known that the Lax-Wendroff scheme gives rise to oscillations, i.e., the Gibb's phenomenon near the shocks. Therefore, high order interpolation methods of the type (4.22)-(4.23) can lead to oscillatory solutions. This suggests that we must use some nonlinear limiter type functions to suppress the oscillations. We notice that our first order scheme is positivity preserving and non-oscillatory. Therefore, in order to achieve a non-oscillatory solution we must switch to the first order scheme in the presence of discontinuities and use the second order interpolation scheme only in smooth regions. This can be achieved with the use of adaptive parameter, say $\chi$ so that in equilibrium or smooth flow regions $\chi \sim 0$ and in discontinuity region $\chi \sim 1$. A possible choice of such a parameter $\chi$ is the switching function of the JST scheme [18] defined by

$$
\chi_{j}^{n}=\frac{\left|p_{j+1}^{n}-2 p_{j}^{n}+p_{j-1}^{n}\right|}{\left|p_{j+1}^{n}+2 p_{j}^{n}+p_{j-1}^{n}\right|} .
$$


Let us denote the right hand sides of (4.22)-(4.23) by $M_{1}^{I I}$ and $M_{2}^{I I}$ respectively and the corresponding first order interpolants by $M_{1}^{I}$ and $M_{2}^{I}$ respectively. Combining both using $\chi$, a second order non-oscillatory interpolation scheme can be obtained as

$$
\begin{aligned}
& M_{1}\left(w^{n}\left(x_{j}+\lambda \Delta t\right)\right)=\chi_{j}^{n} M_{1}^{I}+\left(1-\chi_{j}^{n}\right) M_{1}^{I I} \\
& M_{2}\left(w^{n}\left(x_{j}-\lambda \Delta t\right)\right)=\chi_{j-1}^{n} M_{2}^{I}+\left(1-\chi_{j-1}^{n}\right) M_{2}^{I I} .
\end{aligned}
$$

Note that a different interpolation strategy was employed in the kinetic scheme of $[12,22]$. However, our numerical results confirm the non-oscillatory nature of the interpolating scheme (4.26)-(4.27).

To complete the second order scheme we need to evaluate also the anti-diffusive flux term $\partial \mathcal{D} / \partial x$. Note that the evaluation of $\mathcal{D}$ requires the computation of the slope $\partial w / \partial x$. As explained above, when strong discontinuities such as shocks are present in the solution, this gradient can have very wild variation. This may lead the second order scheme (4.6) to give some unphysical solutions. Therefore, we must apply some nonlinear limiter functions in the calculation of the required gradients. A possible computation of such a slope, which results in an overall non-oscillatory scheme is given by a family of discrete derivatives parametrised by $1 \leq \theta \leq 2$, for example

$$
\frac{\partial w}{\partial x}\left(x_{j}, t^{n}\right)=M M\left(\theta \frac{w_{j+1}^{n}-w_{j}^{n}}{\Delta x}, \frac{w_{j+1}^{n}-w_{j-1}^{n}}{2 \Delta x}, \theta \frac{w_{j}^{n}-w_{j-1}^{n}}{\Delta x}\right) .
$$

Here $M M$ denotes the nonlinear minmod function defined by

$$
M M\left\{v_{1}, v_{2}, \cdots\right\}= \begin{cases}\min _{p}\left\{v_{p}\right\} & \text { if } v_{p}>0 \forall p, \\ \max _{p}\left\{v_{p}\right\} & \text { if } v_{p}<0 \forall p, \\ 0 & \text { otherwise. }\end{cases}
$$

After computing the values of $\mathcal{D}$ at all the mesh points, the derivative $\partial \mathcal{D} / \partial x$ is also calculated using the same minmod recovery procedure. Thus, we have completed the evaluation of all the terms required by the second order scheme (4.6).

\section{Numerical Case Studies}

The new kinetic relaxation scheme is tested on some standard benchmark problems for the Euler equations in one space dimension. In all the problems the computations were carried out on uniform Cartesian grids. In order to avoid the formation of initial and boundary layers in (2.12), the initial and boundary conditions for $f_{k}$ are chosen to be consistent with the equilibrium distribution $M_{k}$. For example, if Dirichlet boundary data are given for the macroscopic variable $w$, say $w=w_{b}$, the initial and boundary conditions for (2.12) are given by

$$
f_{k}(x, t)=M_{k}\left(w_{b}(x, t)\right), f_{k}(x, 0)=M_{k}(w(x, 0)) .
$$

In all computations we have used both the JST switching function as well as $M M$ limiter with $\theta=2$.

Experimental Order of Convergence. Despite the simplicity of the algorithm and operator splitting approach, the kinetic relaxation scheme gives second order convergence. In what follows we test the order of convergence for a smooth solution. We consider an exact periodic solution of the one-dimensional Euler equations

$$
\begin{aligned}
& \rho(x, t)=1.0+0.2 \sin (\pi(x-u t)), \\
& u(x, t)=0.1, p(x, t)=0.5 .
\end{aligned}
$$


The experimental order of convergence (EOC) can be calculated by systematically refining the mesh and examining the behaviour of the global error. Since the exact solution is known, the order of convergence in a certain norm $\|\cdot\|$ can be computed in the following way

$$
\mathrm{EOC}=\log _{2}\left(\frac{\left\|\mathcal{E}_{K / 2}\right\|}{\left\|\mathcal{E}_{K}\right\|}\right)
$$

where $K$ denotes the number of mesh points and $\left\|\mathcal{E}_{K}\right\|$ is a suitable norm of the global error, for example,

$$
\begin{aligned}
\left\|\mathcal{E}_{K}\left(t^{n}\right)\right\|_{L^{1}} & =\Delta x \sum_{j=1}^{K}\left|\rho\left(x_{j}, t^{n}\right)-\rho_{j}^{n}\right|, \\
\left\|\mathcal{E}_{K}\left(t^{n}\right)\right\|_{L^{2}} & =\sqrt{\Delta x \sum_{j=1}^{K}\left(\rho\left(x_{j}, t^{n}\right)-\rho_{j}^{n}\right)^{2}}, \\
\left\|\mathcal{E}_{K}\left(t^{n}\right)\right\|_{L^{\infty}} & =\max _{1 \leq j \leq K}\left|\rho\left(x_{j}, t^{n}\right)-\rho_{j}^{n}\right| .
\end{aligned}
$$

Note that we have used only the density to compute errors. The computational domain $[0,2]$ is consecutively divided into $20,40, \ldots, 2560$ cells. The final time was taken to be $t=0.5$. The table 1 shows the experimental order of convergence computed in the $L^{1}, L^{2}$ and $L^{\infty}$ norms. From the table it is evident that the order of convergence is 2 .

\begin{tabular}{|r|c|c|c|c|c|c|}
\hline$K$ & $L^{1}$ error & EOC & $L^{2}$ error & EOC & $L^{\infty}$ error & EOC \\
\hline \hline 20 & 0.03071610 & & 0.02523467 & & 0.03311415 & \\
\hline 40 & 0.00806604 & 1.929063 & 0.00646948 & 1.963686 & 0.00914183 & 1.856893 \\
\hline 80 & 0.00197558 & 2.029584 & 0.00154233 & 2.068538 & 0.00212876 & 2.102470 \\
\hline 160 & 0.00047793 & 2.047405 & 0.00037085 & 2.056204 & 0.00048072 & 2.146745 \\
\hline 320 & 0.00011763 & 2.022543 & 0.00009145 & 2.019781 & 0.00012036 & 1.997841 \\
\hline 640 & 0.00002922 & 2.009228 & 0.00002277 & 2.005849 & 0.00003281 & 1.875149 \\
\hline 1280 & 0.00000726 & 2.008915 & 0.00000566 & 2.008260 & 0.00000867 & 1.920032 \\
\hline 2560 & 0.00000179 & 2.020010 & 0.00000140 & 2.015375 & 0.00000223 & 1.958988 \\
\hline \hline
\end{tabular}

TABLE 1. $L^{1}, L^{2}$ and $L^{\infty}$ errors with experimental order of convergence for a smooth periodic test case.

Sod Shock Tube Problem. We consider the Sod shock tube problem. The solution consists of a left rarefaction, a contact discontinuity and a right shock. The initial data reads

$$
(\rho, u, p)(x, 0)= \begin{cases}(1.0,0.0,1.0), & \text { if } \quad 0<x<0.5, \\ (0.125,0.0,0.1), & \text { if } \quad 0.5<x<1 .\end{cases}
$$

The computations are done with both the first order and second order schemes on 400 mesh points with a CFL number 0.9. Figure 2 shows the density, velocity and pressure at time $t=0.2$. The results of first order scheme are highly smeared due the excess amount of numerical diffusion. The second order scheme is comparatively much less dissipative and it resolves the discontinuities very well. 

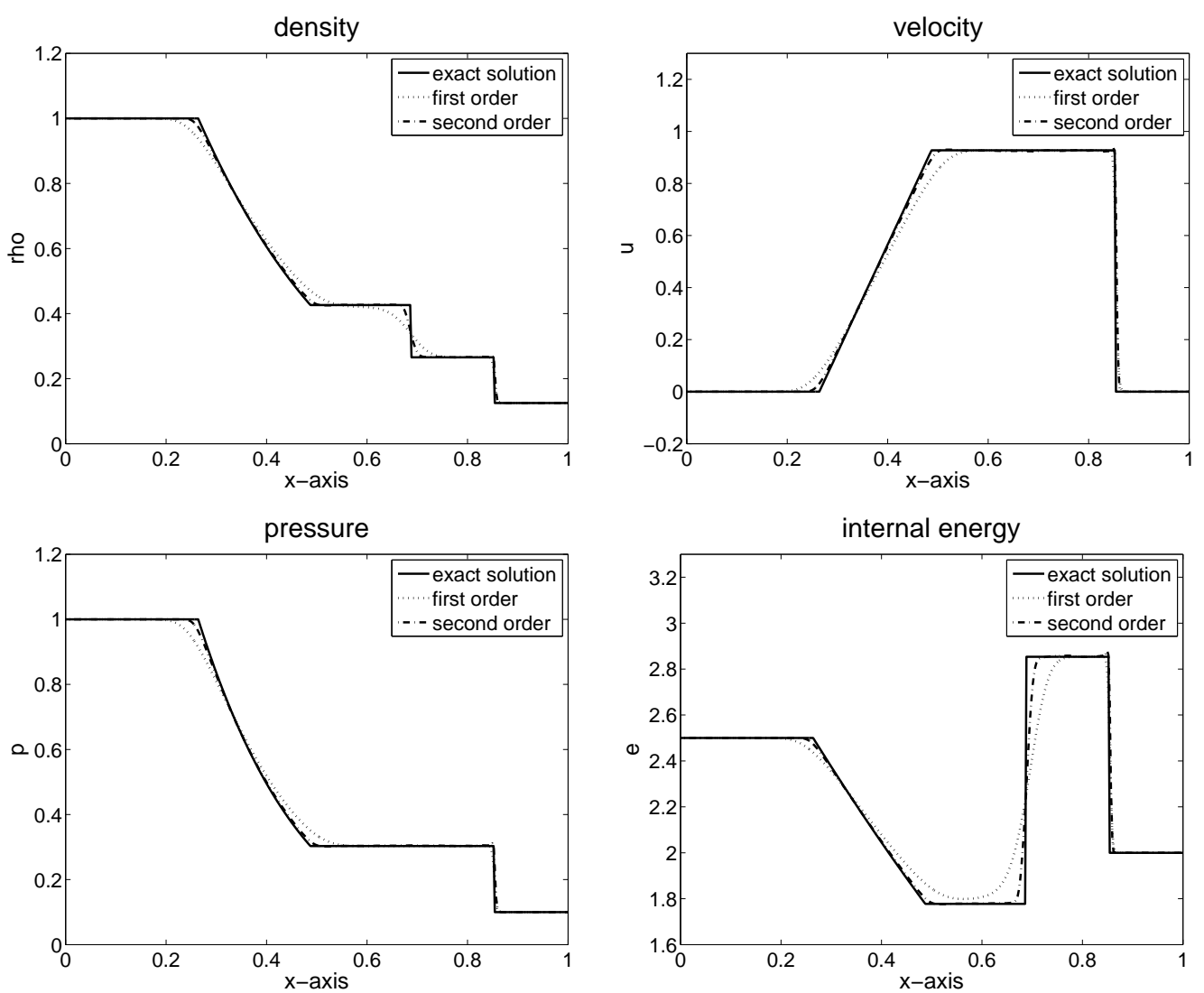

Figure 2. Sod shock tube problem results at $t=0.2$.

Lax Shock Tube Problem. This test case is the Lax shock tube problem. The initial data is given by

$$
(\rho, u, p)(x, 0)= \begin{cases}(0.445,0.698,3.528), & \text { if } \quad 0 \leq x<0.5, \\ (0.5,0.0,0.571), & \text { if } \quad 0.5<x \leq 1 .\end{cases}
$$

We have used used 400 mesh points for the computations and the CFL number was set to 0.9. In Figure 3 we give the plots of density, velocity and pressure at time $t=0.13$. The plots show that the second order scheme gives a sharper resolution of both shocks and expansions.

Strong Rarefactions Riemann Problem. We consider the Riemann problem with initial data

$$
(\rho, u, p)(x, 0)= \begin{cases}(1.0,-0.2,0.4), & \text { if } 0 \leq x<0.5, \\ (1.0,2.0,0.4), & \text { if } 0.5<x \leq 1 .\end{cases}
$$

This is a very difficult problem for many methods because a near vacuum state is reached and failure can occur as a result of negative densities or pressures. For instance, linearised Riemann solvers can fail by giving negative pressures or densities in one or more of the intermediate states for very strong rarefactions, see [14] for a detailed study. In Figure 4 we give the plots of density and pressure at $t=0.15$ computed using first and second order schemes using a grid with 400 mesh points. From the figure we can notice that both the schemes preserve the positivity of density and pressure. 

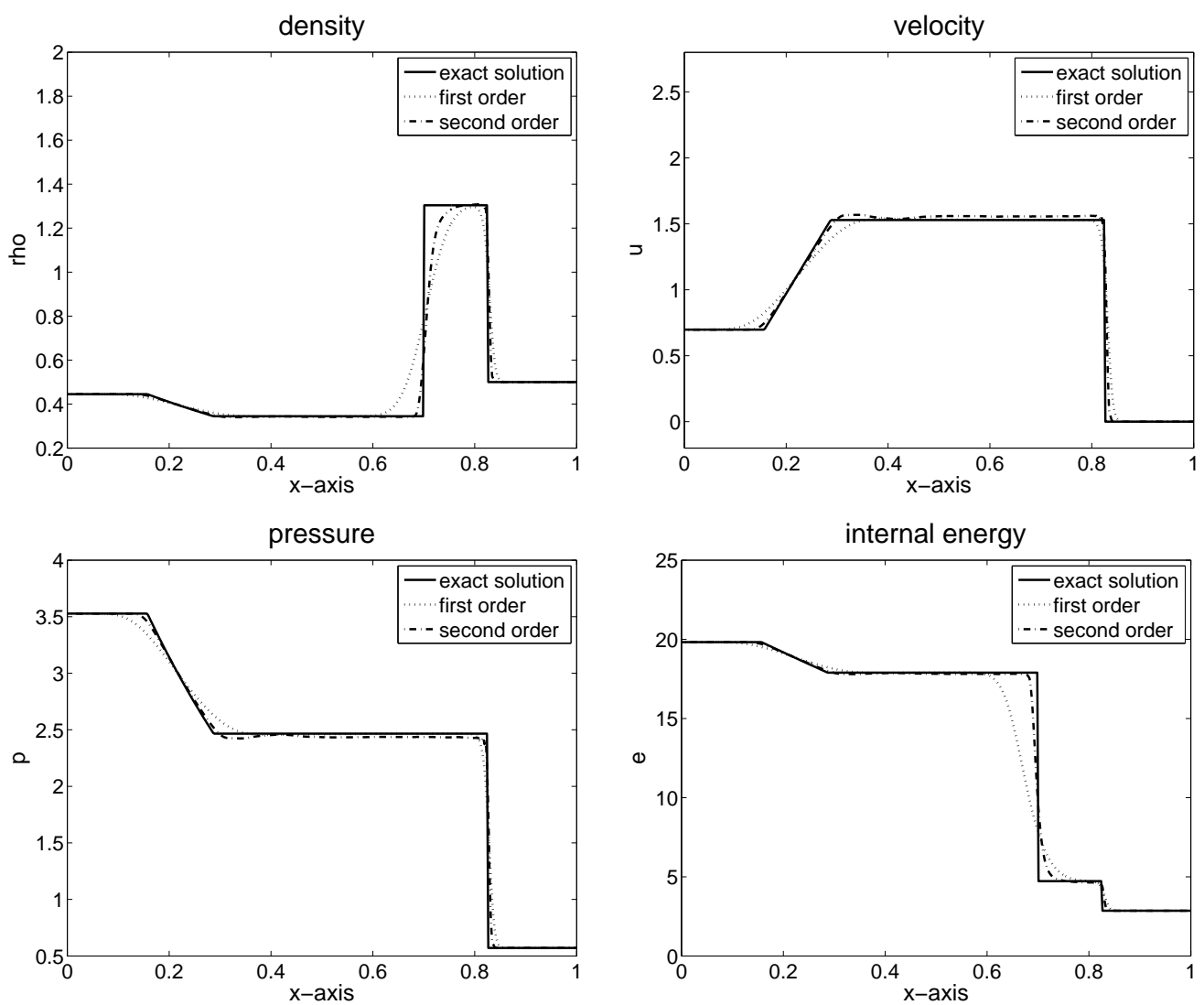

Figure 3. Lax shock tube problem results at $t=0.2$.
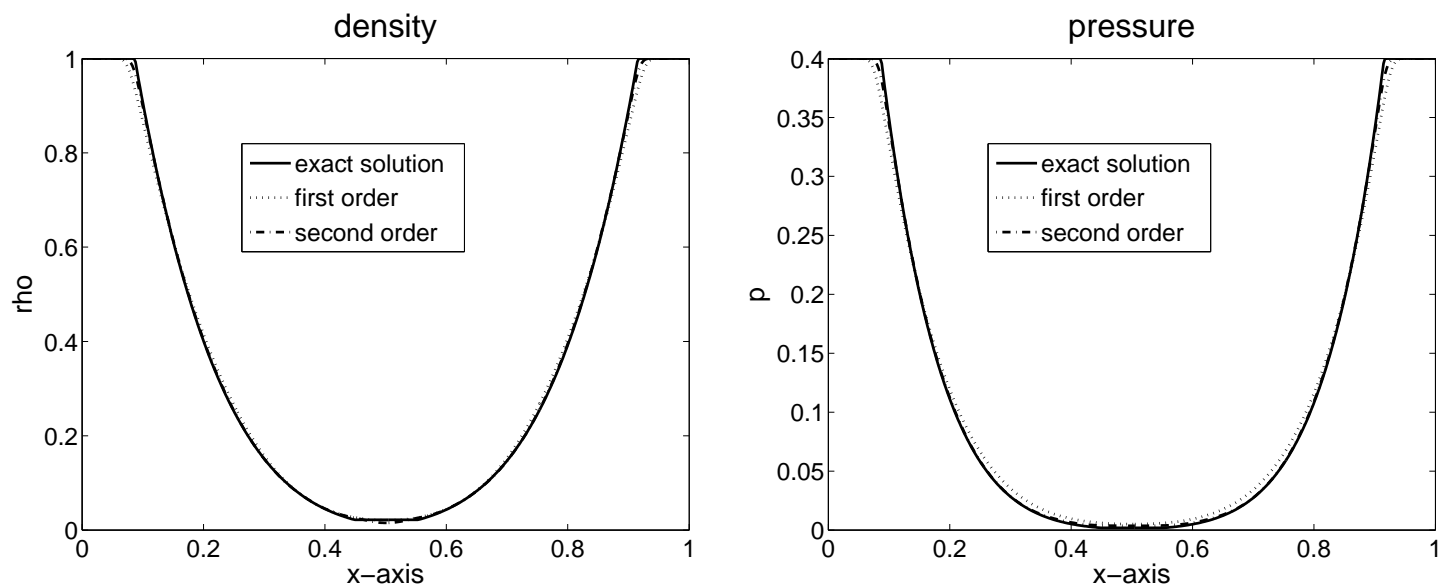

Figure 4. Two strong rarefactions test. The plots of density and pressure at $t=0.15$.

Shock Entropy Wave Interaction. This test problem is taken from [41]. It describes of the interaction of a sinusoidal density perturbation and a supersonic shock wave. A Mach 3 shock wave runs into a smooth acoustic wave, which gets amplified and has a higher frequency behind the shock. 
The initial data reads

$$
(\rho, u, p)(x, 0)= \begin{cases}(3.857143,2.629369,10.333333), & \text { if }-5 \leq x<-4, \\ (1+0.2 \sin (5 x), 0,1), & \text { if }-4 \leq x \leq 5 .\end{cases}
$$

We run the computations on a fine mesh with 1000 points. We use extrapolation boundary conditions at both ends. The CFL number is 0.9 and final time is set to $t=1.8$. In order to compare the results, we have computed the reference solution by running the second order scheme on 4000 mesh points. The results are given in Figure 5. The first order results are extremely smeared despite the use of a fine mesh. However, the second order scheme resolves the flow features quite well.
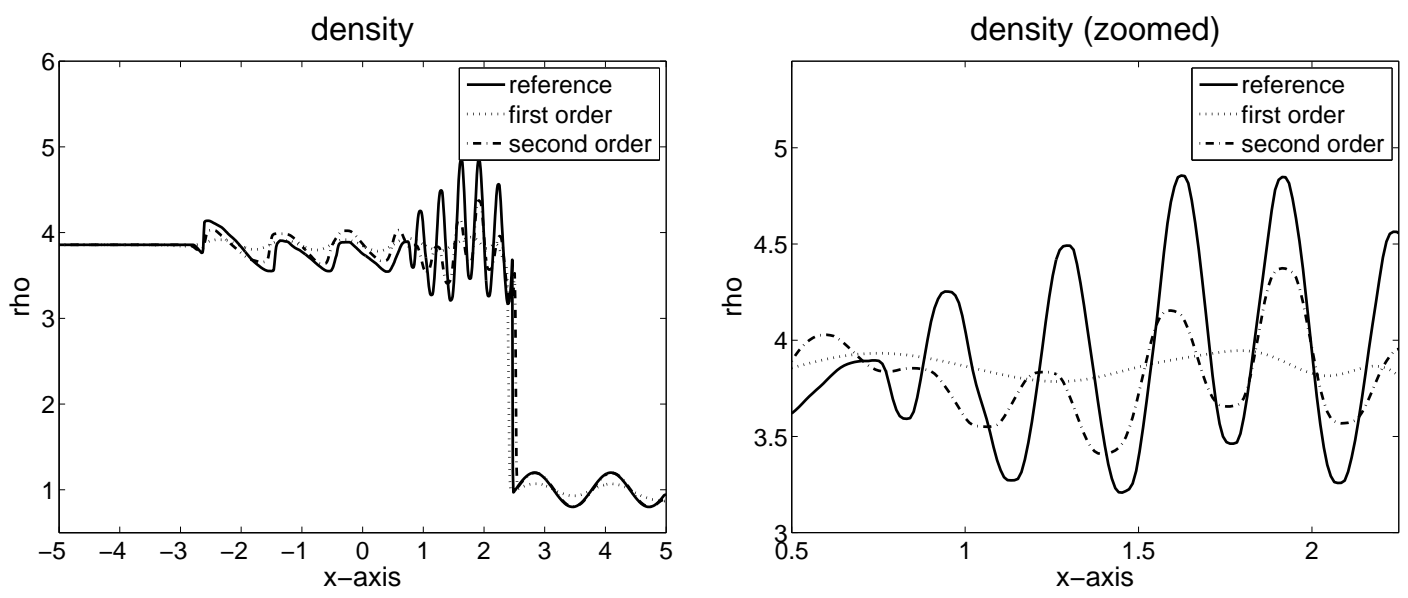

FIGURE 5. The results of shock-acoustic wave interaction problem results at $t=1.8$.

\section{Concluding Remarks}

In this paper a novel upwind kinetic relaxation scheme is developed based on a discrete velocity Boltzmann relaxation system. The first order accurate scheme preserves the positivity of mass density and pressure and is entropy stable. The second order method involves the use of an antidiffusive Chapman-Enskog distribution function. The present method involves only interpolation and the use of limiters and therefore it is different from the conventional numerical methods. The kinetic relaxation scheme retains many attractive features of central schemes, such as neither Riemann solvers nor characteristic decompositions are needed. Both the first order and second order schemes are stable up to a CFL number 1.0. The scheme is tested on some benchmark problems for Euler equations and the results demonstrate its robustness and efficiency in capturing the flow features accurately. Generalisation to multi-dimensions can be done e.g. by directional splitting or using theory of bicharacteristics and applying the scheme for one-dimensional wave propagation along a particular bicharacteristic direction, e.g. $(\cos \theta, \sin \theta), \theta=\pi / 4,3 \pi / 4,5 \pi / 4,7 \pi / 4$.

\section{ACKNOWLEDGEMENT}

This work was initiated when ML was visiting the Department of Mathematics, Indian Institute of Science, Bangalore. We thank the Department of Science and Technology (DST), Government of India and Deutscher Akademischer Austausch Dienst (DAAD) for providing financial support for our joint work. KRA's research is funded by the Council of Scientific and Industrial Research (CSIR) under the grant-09/079(2084)/2006-EMR-1. PP is supported by the Department of Atomic Energy, 
Government of India under Raja Ramanna fellowship. These authors gratefully acknowledge their respective grants.

\section{REFERENCES}

[1] D. Aregba-Driollet and R. Natalini. Discrete kinetic schemes for multidimensional systems of conservation laws. SIAM J. Numer. Anal., 37:1973-2004, 2000.

[2] S. Balasubramanyam and S. V. Raghurama Rao. A grid-free upwind relaxation scheme for inviscid compressible flows. Internat. J. Numer. Methods Fluids, 51:159-196, 2006.

[3] P. L. Bhatnagar, E. P. Gross, and M. Krook. A model for collision processes in gases. I. Small amplitude processes in charged and neutral one-component systems. Phys. Rev., 94:511-525, 1954.

[4] F. Bouchut. Construction of BGK models with a family of kinetic entropies for a given system of conservation laws. J. Statist. Phys., 95:113-170, 1999.

[5] F. Bouchut. Stability of relaxation models for conservation laws. In European Congress of Mathematics, pages 95-101. Eur. Math. Soc. Zürich, 2005.

[6] C. Cercignani. The Boltzmann equation and its applications, volume 67 of Applied Mathematical Sciences. Springer Verlag, New York, 1988.

[7] C. Cercignani, R. Illner, and M. Pulvirenti. The mathematical theory of dilute gases, volume 106 of Applied Mathematical Sciences. Springer Verlag, New York, 1994.

[8] S. Chapman and T. G. Cowling. The mathematical theory of non-uniform gases. Cambridge University Press, 1939.

[9] G. Q. Chen, C. D. Levermore, and T.-P. Liu. Hyperbolic conservation laws with stiff relaxation terms and entropy. Comm. Pure Appl. Math., 47:787-830, 1994.

[10] F. Coquel and B. Perthame. Relaxation of energy and approximate Riemann solvers for general pressure laws in fluid dynamics. SIAM J. Numer. Anal., 35:2223-2249, 1998.

[11] S. M. Deshpande. On the Maxwellian distribution, symmetric form, and entropy conservation for the Euler equations. Technical Report 2583, NASA, Langley, 1986.

[12] S. M. Deshpande. A second order accurate, kinetic-theory based, method for inviscid compressible flows. Technical Report 2613, NASA, Langley, 1986.

[13] S. M. Deshpande. Kinetic flux splitting schemes. In M. Hafez and K. Oshima, editors, Computational Fluid Dynamics Review 1995: a State-of-the-art Reference to the Latest Developments in CFD. Wiley, 1995.

[14] B. Einfeldt, C.-D. Munz, P. L. Roe, and B. Sjögreen. On Godunov-type methods near low densities. J. Comput. Phys., 92:273-295, 1991.

[15] J. L. Estivalezes and P. Villedieu. High-order positivity-preserving kinetic schemes for the compressible Euler equations. SIAM J. Numer. Anal., 33:2050-2067, 1996.

[16] E. Godlewski and P.-A. Raviart. Numerical approximation of hyperbolic systems of conservation laws, volume 118 of Applied Mathematical Sciences. Springer-Verlag, New York, 1996.

[17] C. Hirsch. Numerical computation of internal and external flows (vol2: Computational methods for inviscid and viscous flows). John Wiley and Sons, New York, 1990.

[18] A. Jameson, W. Schmidt, and E. Turkel. Numerical solution of the euler equations by finite volume methods using Runge Kutta time stepping schemes. AIAA Paper 81-1259, 1981.

[19] S. Jin. Runge-Kutta methods for hyperbolic conservation laws with stiff relaxation terms. J. Comput. Phys., 122:51-67, 1995.

[20] S. Jin and Z. P. Xin. The relaxation schemes for systems of conservation laws in arbitrary space dimensions. Comm. Pure Appl. Math., 48:235-276, 1995.

[21] M. A. Katsoulakis, G. Kossioris, and C. Makridakis. Convergence and error estimates of relaxation schemes for multidimensional conservation laws. Comm. Partial Differential Equations, 24:395-424, 1999.

[22] M. Kunik, S. Qamar, and G. Warnecke. Second-order accurate kinetic schemes for the ultra-relativistic Euler equations. J. Comput. Phys., 192:695-726, 2003.

[23] R. J. LeVeque. Finite Volume Methods for Hyperbolic Problems. Cambridge Texts in Applied Mathematics. Cambridge University Press, Cambridge, 2002.

[24] T.-P. Liu. Hyperbolic conservation laws with relaxation. Comm. Math. Phys., 108:153-175, 1987.

[25] I. Müller and T. Ruggeri. Rational Extended Thermodynamics, volume 37 of Springer Tracts in Natural Philosophy. Springer Verlag, New York, 1998.

[26] R. Natalini. Recent results on hyperbolic relaxation problems. In Analysis of systems of conservation laws (Aachen, 1997), volume 99 of Chapman \& Hall/CRC Monogr. Surv. Pure Appl. Math., pages 128-198. Chapman \& Hall/CRC, Boca Raton, FL, 1999. 
[27] H. Nessyahu and E. Tadmor. Nonoscillatory central differencing for hyperbolic conservation laws. J. Comput. Phys., 87:408-463, 1990.

[28] S. Noelle. The MoT-ICE: a new high-resolution wave-propagation algorithm for multidimensional systems of conservation laws based on Fey's method of transport. J. Comput. Phys., 164:283-334, 2000.

[29] L. Pareschi and G. Russo. Implicit-Explicit Runge-Kutta schemes and applications to hyperbolic systems with relaxation. J. Sci. Comput., 25:129-155, 2005.

[30] R. B. Pember. Numerical methods for hyperbolic conservation laws with stiff relaxation. II. Higher-order Godunov methods. SIAM J. Sci. Comput., 14:824-859, 1993.

[31] B. Perthame. Boltzmann type schemes for gas dynamics and the entropy property. SIAM J. Numer. Anal., 27:1405-1421, 1990.

[32] B. Perthame. Second-order Boltzmann schemes for compressible Euler equations in one and two space dimensions. SIAM J. Numer. Anal., 29:1-19, 1992.

[33] T. Płatkowski and R. Illner. Discrete velocity models of the Boltzmann equation: a survey on the mathematical aspects of the theory. SIAM Rev., 30:213-255, 1988.

[34] D. I. Pullin. Direct simulation methods for compressible inviscid ideal-gas flow. J. Comput. Phys., 34:231-244, 1980.

[35] S. V. Raghurama Rao. New numerical schemes based on relaxation systems for conservation laws. Berichte der Arbeitsgruppe Technomathematik 249, Technische Universität Kaiserslautern, 2002.

[36] S. V. Raghurama Rao and K. Balakrishna. An accurate shock capturing algorithm with a relaxation system for hyperbolic conservation laws, AIAA-2003-4115. In 16th AIAA Computational Fluid Dynamics Conference, Orlando, Florida, June 23-26 2003. American Institute of Aeronautics and Astronautics.

[37] S. V. Raghurama Rao and S. M. Deshpande. Peculiar velocity based upwind methods for compressible flows. In Recent advances in fluid mechanics, pages 17-33. Gordon and Breach, Amsterdam, 1998.

[38] S. V. Raghurama Rao and M. Subba Rao. A simple multidimensional relaxation scheme based on characteristics and interpolation, AIAA-2003-3535. In 16th AIAA Computational Fluid Dynamics Conference, Orlando, Florida, June 23-26 2003. American Institute of Aeronautics and Astronautics.

[39] R. H. Sanders and K. H. Prendergast. The possible relation of the 3-KILOPARSEC arm to explosions in the galactic nucleus. Astrophys. J., 188:489-500, 1974.

[40] D. Serre. Relaxations semi-linéaire et cinétique des systèmes de lois de conservation. Ann. Inst. H. Poincaré Anal. Non Linéaire, 17:169-192, 2000.

[41] C.-W. Shu and S. Osher. Efficient implementation of essentially non-oscillatory shock-capturing schemes II. J. Comput. Phys., 83:32-78, 1989.

[42] E. Tadmor. Approximate solutions of nonlinear conservation laws. In Advanced numerical approximation of nonlinear hyperbolic equations (Cetraro, 1997), volume 1697 of Lecture Notes in Math., pages 1-149. Springer, Berlin, 1998.

[43] E. F. Toro. Riemann solvers and numerical methods for fluid dynamics. Springer-Verlag, Berlin, second edition, 1999. A practical introduction.

[44] J. Wang and G. Warnecke. Convergence of relaxing schemes for conservation laws. In Advances in nonlinear partial differential equations and related areas (Beijing, 1997), pages 300-325. World Sci. Publ., River Edge, NJ, 1998.

[45] G. B. Whitham. Linear and Nonlinear Waves. John Wiley, New York, 1974.

[46] K. Xu. Gas kinetic scheme for unsteady compressible flow simulations. In Lecture Note Series 1998-03. Von Kárman Institute for Fluid Dynamics, Rhode Saint Genèse, Belgium, 1998.

[47] K. Xu. Gas evolution dynamics in Godunov-type schemes and analysis of numerical shock instability. Technical Report 99-6, Institute for Computer Applications in Science and Engineering (ICASE), 1999. 
Department of Mathematics, Indian Institute of Science, Bangalore 560012, India

E-mail address: arunkr@math.iisc.ernet.in

Institute of Mathematics, University of Mainz, D-55099 Mainz, Germany

E-mail address: lukacova@uni-mainz.de

URL: http://www . mathematik. uni-mainz/Members/lukacova

Department of Mathematics, Indian Institute of Science, Bangalore 560012, India

E-mail address: prasad@math.iisc.ernet.in

$U R L:$ http://math.iisc.ernet.in/ ${ }^{\sim}$ prasad

Department of Aerospace Engineering, Indian Institute of Science, Bangalore 560012, India E-mail address: raghu@aero.iisc.ernet.in 